\title{
Threshold Saturation of Spatially-Coupled Codes on Intersymbol-Interference Channels
}

\author{
Phong S. Nguyen, Arvind Yedla, Henry D. Pfister, and Krishna R. Narayanan \\ Department of Electrical and Computer Engineering, Texas A\&M University \\ \{psn, yarvind, hpfister, krn\}@tamu.edu
}

\begin{abstract}
Recently, it has been observed that terminated lowdensity-parity-check (LDPC) convolutional codes (or spatiallycoupled codes) appear to approach the capacity universally across the class of binary memoryless channels. This is facilitated by the "threshold saturation" effect whereby the belief-propagation (BP) threshold of the spatially-coupled ensemble is boosted to the maximum a-posteriori (MAP) threshold of the underlying constituent ensemble.

In this paper, we consider spatially-coupled codes over intersymbol-interference (ISI) channels under joint iterative decoding where we empirically show that threshold saturation also occurs. This can be observed by first identifying the GEXIT curve that naturally obeys the general area theorem. From this curve, the corresponding MAP and the BP threshold estimates are then numerically obtained. Given the fact that regular LDPC codes can achieve the symmetric information rate (SIR) under MAP decoding, we conjecture that spatially-coupled codes with joint iterative decoding can universally approach the SIR of ISI channels.
\end{abstract}

\section{INTRODUCTION}

LDPC convolutional codes, which were introduced in [1] and shown to have excellent BP thresholds in [2], have recently been observed to universally approach the capacity of various memoryless channels. The fundamental mechanism behind this is explained well in [3], where it is proven analytically for the BEC that the BP threshold of a particular spatially-coupled ensemble converges to the maximum a-posteriori (MAP) threshold of the underlying ensemble. A similar result was also observed independently in [4] and stated as a conjecture. Such a phenomenon is now called "threshold saturation via spatial coupling" and has also been empirically observed for general binary-input symmetric-output memoryless (BMS) channels [5]. In fact, threshold saturation seems to be quite general and has now been observed in a wide range of problems.

In the realm of channels with memory and particularly intersymbol interference (ISI) channels, the capacity may not be achievable via equiprobable signaling. For linear codes, a popular practice is to compare instead with the symmetric information rate (SIR), which is also known as $C_{\text {i.u.d. }}$ [6], because this rate is achievable by random linear codes with maximum-likelihood (ML) decoding. For LDPC codes over ISI channels, a joint iterative BP decoder that operates on a large graph representing both the channel and the code constraints [6], [7] can perform quite well. Progress has also been made on the design of SIR-approaching irregular LDPC

This material is based upon work supported by the National Science Foundation under Grant No. 0747470. The work of P. Nguyen was also supported in part by a Vietnam Education Foundation fellowship. Any opinions, findings, conclusions, or recommendations expressed in this material are those of the authors and do not necessarily reflect the views of the National Science Foundation. codes for some specific ISI channels [8], [9], [10], [11], [12]. However, channel parameters must be known at the transmitter for such designs and therefore universality appears difficult to achieve.

Since spatially-coupled codes and the threshold saturation effect have now shown benefits in many communication problems, it is quite natural to consider them as a potential candidate to universally approach the SIR of ISI channels with low decoding complexity. In fact, the combination of spatiallycoupled codes and ISI channels was recently considered by Kudekar and Kasai [13] for the simple dicode erasure channel (DEC) from [12], [14]. They provided a numerical evidence that the joint BP threshold of the spatially coupled codes can approach the SIR over the DEC. However, the EXITlike curves they considered were not equipped with an area theorem and therefore could not be directly connected with the MAP threshold of the underlying ensemble. Thus, the threshold saturation effect was only indirectly observed.

In this paper, we first focus on the case of general ISI channels where, by deriving the appropriate GEXIT curve and associated area theorem, the MAP threshold upper bound can be computed and threshold saturation can be seen. As a consequence, it is possible for spatially-coupled codes to closely approach the SIR of ISI channels under joint iterative BP decoding because regular LDPC codes can achieve the SIR under MAP decoding [15]. Also, we revisit the transmission of the spatially-coupled codes over the DEC as a special case. For this channel, with the MAP threshold estimated from the (G)EXIT curve, threshold saturation can also be observed to occur exactly. Furthermore, the simplicity of the DEC allows us to provide a rigorous analysis of the upper bound on the MAP threshold.

\section{BACKGROUND}

\section{A. ISI Channels and the SIR}

Let the input alphabet $\mathcal{X}$ be finite, $\left\{X_{i}\right\}_{i \in \mathbb{Z}}$ be the discretetime input sequence (i.e., $X_{i} \in \mathcal{X}$ ) and $\left\{Y_{i}\right\}_{i \in \mathbb{Z}}$ be the discretetime output sequence with $Y_{i} \in \mathbb{R}$. Many ISI channels of interest can be modeled by $Y_{i}=\sum_{t=0}^{\nu} a_{t} X_{i-t}+N_{i}$, where the channel memory is $\nu,\left\{a_{t}\right\}_{t=0}^{\nu}$ is the set of tap coefficients and $\left\{N_{i}\right\}_{i \in \mathbb{Z}}$ is a sequence of independent noise random variables. One can also write the above as $Y_{i}=Z_{i}+N_{i}$ where $Z_{i}=\sum_{t=0}^{\nu} a_{t} X_{i-t}$ is the ISI channel output without noise. In this paper, we restrict ourselves to the class of binary-input ISI channels. Often, the tap coefficients are represented through a transform domain polynomial $a(D)=\sum_{t=0}^{\nu} a_{t} D^{t}$.

The subject of Section IV is the dicode erasure channel (DEC), which is basically a 1st-order differentiator whose 


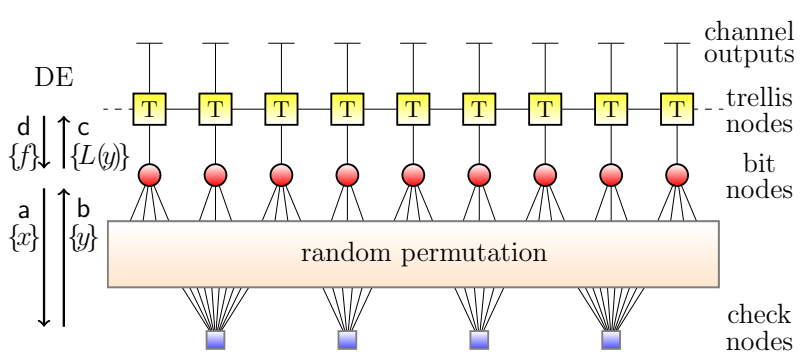

Figure 1. Gallager-Tanner-Wiberg graph of the joint BP decoder for ISI channels. The notations $a, b, c, d$ denote the average densities of the messages traversing along the graph used in density evolution (DE). The quantities inside the brackets are erasure rates used in DE for the DEC case.

output is erased with probability $\epsilon$ and transmitted perfectly with probability $1-\epsilon$. Meanwhile, Section III considers more general ISI channels among which the most common is linear ISI channels with additive white Gaussian noise (AWGN). Unfortunately, no closed-form solutions for the SIR are known in this case. Instead, the numerical method described in [16], [17] is typically used to give tight estimates of the SIR.

\section{B. LDPC Ensembles and the Joint BP Decoder}

The standard irregular LDPC ensemble is characterized by its degree distribution (d.d.), which represents the fraction of nodes (or edges) of particular degrees [18]. From the edge perspective, the d.d. pair consists of two polynomials $\lambda(x)=$ $\sum_{i \geq 1} \lambda_{i} x^{i-1}$ and $\rho(x)=\sum_{i \geq 1} \rho_{i} x^{i-1}$. The LDPC ensemble can also be viewed from the node perspective where its d.d. pair $L(x)=\sum_{i \geq 1} L_{i} x^{i}$ and $R(x)=\sum_{i \geq 1} R_{i} x^{i}$. The design rate of an LDPC ensemble is given by $r=1-L^{\prime}(1) / R^{\prime}(1)$.

When LDPC codes are transmitted over the ISI channel, one can construct a large graph by joining the code graph and the channel graph together as depicted in Fig. 1. Working on this joint graph, a joint iterative decoder typically passes the information back and forth between the channel detector and the LDPC decoder. This technique is termed as turbo equalization and was first considered by Douillard et al. in the context of turbo codes [19]. For analysis, we also require the addition of a random scrambling vector to symmetrize the effective channel [20]. This is very similar to using a random coset of the LDPC code to allow analysis of the decoder using the all-zero codeword assumption; this technique was also used in [6] where they proved a concentration theorem and derived the density evolution (DE) equations for ISI channels.

\section{Spatially-Coupled Ensembles}

The class of spatially-coupled ensembles can be defined quite broadly. In this paper, we mainly consider two basic variants (see details in [3]) as discussed below.

1) The $(l, r, L)$ ensemble: The $(l, r, L)$ spatially-coupled ensemble (with $l$ odd so that $\hat{l}=\frac{l-1}{2} \in \mathbb{N}$ ) can be constructed from the underlying $(l, r)$-regular LDPC ensemble. At each position from $[1, L]$ one has $M$ bit nodes and $\frac{l}{r} M$ check nodes just like in the $(l, r)$-regular case. However, each bit node at position $i$ is connected to one check node at each position from $i-\hat{l}$ to $i+\hat{l}$. In doing this, one also needs to add $\frac{l}{r} M$ extra check nodes at each of $\hat{l}$ extra positions on each side. For example, a joint code/channel graph for the $(3,6, L)$ ensemble and the ISI channels is shown in Fig. 2.

2) The $(l, r, L, w)$ ensemble: The $(l, r, L, w)$ can be obtained with the introduction of a "smoothing" parameter $w$. One still places $M$ variable nodes at each position in $[1, L]$ but places $\frac{l}{r} M$ check nodes at each position in $[1, L+w-1]$. Each bit node at position $i$ is connected uniformly and independently to a total of $l$ check nodes at positions from the range $[i, i+w-1]$.

\section{General ISI Channels}

The main part of this paper focuses on ISI channels with general noise models. The MAP upper bound for general binary memoryless symmetric channels was presented by Méasson et al. and conjectured to be tight [21]. For general ISI channels, we apply a similar technique to give an estimate of the MAP threshold of the underlying ensemble by first constructing the BP-GEXIT curve that follows an area theorem. The BP thresholds of the coupled ensembles are then computed via DE and the threshold saturation effect is observed. In addition, simulations on the performance of the joint BP decoder for coupled codes of finite length are conducted to validate these thresholds.

\section{A. GEXIT Curves for the ISI channels}

When the channel input $X_{1}^{n} \triangleq\left(X_{1}, X_{2}, \ldots, X_{n}\right)$ is chosen uniformly at random from a suitable binary linear code ${ }^{1}$, the ISI output without noise $Z_{i}$ at some index $i$ is a discrete random variable characterized by its probability mass function $p_{Z_{i}}(z)$ for all $z$ in the alphabet $\mathcal{Z}$. For example, in the case of a dicode channel, $\mathcal{Z}=\{0,+2,-2\}$ and $p_{Z_{i}}(0)=\frac{1}{2}, p_{Z_{i}}(+2)=$ $p_{Z_{i}}(-2)=\frac{1}{4}$. The channel from $Z_{i}$ to $Y_{i}$ is a $|\mathcal{Z}|$-ary input memoryless channel characterized by its transition probability density $p_{Y_{i} \mid Z_{i}}(y \mid z)$. Without specifying the index, we denote $\mathrm{h} \triangleq H(Z \mid Y)$ and get

$$
\mathrm{h}=H(Z)-\int_{-\infty}^{\infty} \sum_{z} p(z) p(y \mid z) \log _{2}\left\{\frac{p(y \mid z)}{\sum_{z^{\prime}} p\left(z^{\prime}\right) p\left(y \mid z^{\prime}\right)}\right\} \mathrm{d} y .
$$

Instead of looking at a particular channel, we assume that the channel from $Z_{i}$ to $Y_{i}$ is from a smooth family $\left\{\mathcal{M}\left(\mathrm{h}_{i}\right)\right\}_{\mathrm{h}_{i}}$ of $|\mathcal{Z}|$-ary input memoryless channels characterized by conditional entropy $h_{i}$. A further assumption is made that all individual channel families are parameterized in a smooth way by a common parameter ${ }^{2} \epsilon$, i.e., $\mathrm{h}_{i}=H\left(Z_{i} \mid Y_{i}\right)(\epsilon)$.

With the convention that $y_{\sim i} \triangleq y_{1}^{n} \backslash y_{i}$, define $\phi_{i}\left(y_{\sim i}\right) \triangleq$ $\left\{P_{Z_{i} \mid Y_{\sim i}}\left(z \mid y_{\sim i}\right): z \in \mathcal{Z}\right\}$ and the random vector $\Phi_{i} \triangleq \phi_{i}\left(Y_{\sim i}\right)$. Each value of $\phi_{i}$ is a vector of length $|\mathcal{Z}|$ in the $(|\mathcal{Z}|-1)$ dimensional probability simplex. The index of the vector associated with $z \in \mathcal{Z}$ is denoted by $[z]$. One can see that $\Phi_{i}$ is a sufficient statistic for estimating $Z_{i}$ given $Y_{\sim i}$, i.e., $Z_{i} \rightarrow \Phi_{i}\left(Y_{\sim i}\right) \rightarrow Y_{\sim i}$ forms a Markov chain ${ }^{3}$.

\footnotetext{
${ }^{1}$ The code is proper [18, p. 14] and its dual code contains no codewords involving only 0 's and a run of $(\nu+1) 1$ 's.

${ }^{2}$ For the AWGN case, a convenient choice for $\epsilon$ is $\epsilon=-\frac{1}{2 \sigma^{2}}$.

${ }^{3}$ One way to see this is to write

$P_{Y_{\sim i} \mid Z_{i}}\left(y_{\sim i} \mid z_{i}\right)=\frac{P_{Z_{i} \mid Y_{\sim i}}\left(z_{i} \mid y_{\sim i}\right)}{P_{Z_{i}}\left(z_{i}\right)} P_{Y_{\sim i}}\left(y_{\sim i}\right)=\frac{\Phi_{i} \cdot e_{\left[z_{i}\right]}^{T}}{P_{Z_{i}}\left(z_{i}\right)} P_{Y_{\sim i}}\left(y_{\sim i}\right)$,
}

where $e_{[z]}^{T}$ is the standard basis column vector with a 1 in the index $[z]$, and apply the result from [18, p. 29]. 


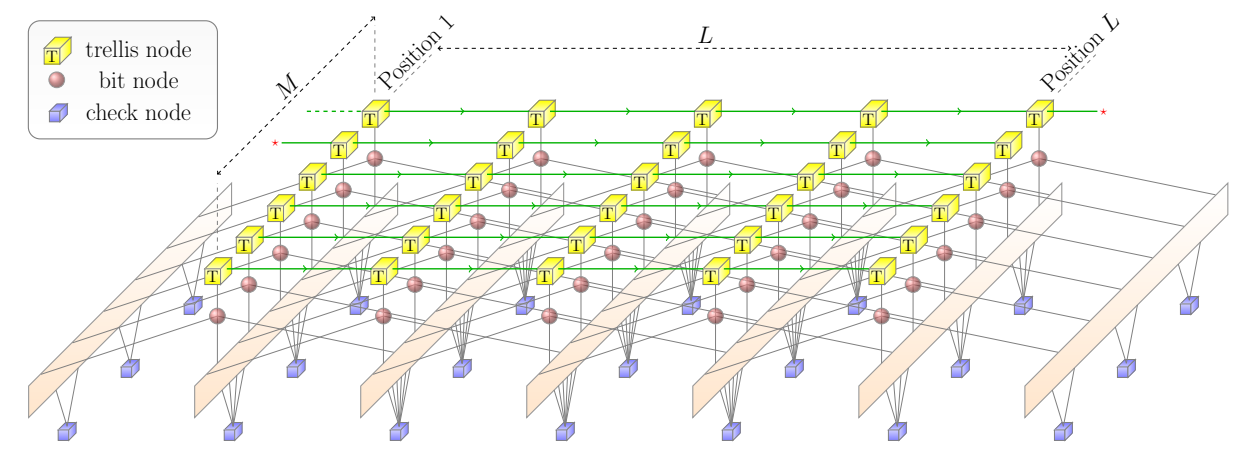

Figure 2. The joint graph for the $(l, r, L)$ ensemble over the ISI channels. Illustrated in this figure is the case where $l=3$ and $r=6$. In the setup we consider, the bit transmission is row by row where the order of transmission within each row is implied by the (green) arrows. The (red) stars are to "connect" consecutive rows.

Definition 1: Suppose the initial state in the trellis is $S_{0}$. Let $X_{1}^{n}$ chosen according to $p_{X_{1}^{n}}\left(x_{1}^{n}\right)$ be the input sequence, $Z_{1}^{n}$ be the ISI output sequence without noise and $Y_{1}^{n}$ be the final channel output sequence, i.e., $Y_{i}$ is the result of transmitting $Z_{i}$ over the smooth family $\left\{\mathcal{M}\left(\mathrm{h}_{i}\right)\right\}_{\mathrm{h}_{i}}$ of memoryless channels. Then the $i$ th GEXIT function is

$$
\mathrm{G}_{i}\left(\mathrm{~h}_{1}, \ldots, \mathrm{h}_{n}\right)=\frac{\partial H\left(X_{1}^{n} \mid Y_{1}^{n}\left(\mathrm{~h}_{1}, \ldots, \mathrm{h}_{n}\right), S_{0}\right)}{\partial \mathrm{h}_{i}}
$$

and the average GEXIT function is defined by $\mathrm{G}\left(\mathrm{h}_{1}, \ldots, \mathrm{h}_{n}\right)=$ $\frac{1}{n} \sum_{i=1}^{n} \mathrm{G}_{i}\left(\mathrm{~h}_{1}, \ldots, \mathrm{h}_{n}\right)$. For the case where all channel families are the same, i.e., $\mathrm{h}_{i}=\mathrm{h}$, we have

$$
\mathrm{G}(\mathrm{h})=\frac{1}{n} \cdot \frac{\mathrm{d} H\left(X_{1}^{n} \mid Y_{1}^{n}(\mathrm{~h}), S_{0}\right)}{\mathrm{dh}} .
$$

Lemma 1: Assume that all the channel families are the same $^{4}$, i.e., $\mathrm{h}_{i}=\mathrm{h}$. Then, the $i$ th GEXIT function is given by $\mathrm{G}_{i}(\mathrm{~h})=\sum_{z} p(z) \int_{v} \mathrm{a}_{i, z}(\underline{v}) \kappa_{i, z}(\underline{v}) \mathrm{d} \underline{v}$ where $\mathrm{a}_{i, z}$ is the distribution of the vector $\Phi_{i}$ given $Z_{i}=z, \underline{v}$ is a vector of length $|\mathcal{Z}|$ in the $(|\mathcal{Z}|-1)$-dimensional probability simplex and the GEXIT kernel (for $i$ and $z$ ) is ${ }^{5}$

$$
\kappa_{i, z}(\underline{v})=\frac{\int_{-\infty}^{\infty} \frac{\partial}{\partial \epsilon} p\left(y_{i} \mid z\right) \log _{2}\left\{\frac{\sum_{z^{\prime}} v_{\left[z^{\prime}\right]} p\left(y_{i} \mid z^{\prime}\right)}{v_{[z]} p\left(y_{i} \mid z\right)}\right\} \mathrm{d} y_{i}}{\int_{-\infty}^{\infty} \sum_{z} p(z) \frac{\partial}{\partial \epsilon} p\left(y_{i} \mid z\right) \log _{2}\left\{\frac{\sum_{z^{\prime}} p\left(z^{\prime}\right) p\left(y_{i} \mid z^{\prime}\right)}{p(z) p\left(y_{i} \mid z\right)}\right\} \mathrm{d} y_{i}} .
$$

Proof: Suppose the initial state is $S_{0}$, we start by writing

$$
\begin{aligned}
H\left(X_{1}^{n} \mid Y_{1}^{n}, S_{0}\right) & =H\left(Z_{1}^{n} \mid Y_{1}^{n}, S_{0}\right) \\
& =H\left(Z_{i} \mid Y_{1}^{n}, S_{0}\right)+H\left(Z_{\sim i} \mid Y_{1}^{n}, Z_{i}, S_{0}\right) .
\end{aligned}
$$

For simplicity of notation, we drop $S_{0}$ in all the expressions although the dependency on $S_{0}$ is always implied. From (1) and (2), it is clear that

We also have

$$
\mathrm{G}_{i}(\mathrm{~h})=\frac{\partial}{\partial \mathrm{h}_{i}} H\left(Z_{i}\left|Y_{1}^{n}\left(\mathrm{~h}_{1}, \ldots, \mathrm{h}_{n}\right)\right|_{\mathrm{h}_{i}=\mathrm{h} \forall i} .\right.
$$

$$
\begin{aligned}
H\left(Z_{i} \mid Y_{1}^{n}\right)= & H\left(Z_{i} \mid Y_{i}, \Phi_{i}\left(Y_{\sim i}\right)\right) \\
= & -\int_{\phi_{i}} \int_{y_{i}} \sum_{z_{i}} p\left(z_{i}\right) p\left(\phi_{i} \mid z_{i}\right) p\left(y_{i} \mid z_{i}\right) . \\
& \cdot \log _{2}\left\{\frac{p\left(z_{i} \mid \phi_{i}\right) p\left(y_{i} \mid z_{i}\right)}{\sum_{z_{i}^{\prime}} p\left(z_{i}^{\prime} \mid \phi_{i}\right) p\left(y_{i} \mid z_{i}^{\prime}\right)}\right\} \mathrm{d} y_{i} \mathrm{~d} \phi_{i}
\end{aligned}
$$

\footnotetext{
${ }^{4}$ Note that for the case of different channel families, one can still compute the $i$ th GEXIT function as a function of the common parameter $\epsilon$.

${ }^{5} p\left(y_{i} \mid z\right)$ is dependent on $\mathrm{h}_{i}$ and hence is dependent on $\epsilon$.
}

where (3) follows from the Bayes' theorem and the fact that

$$
p\left(z_{i}, \phi_{i}, y_{i}\right)=p\left(z_{i}, \phi_{i}\right) p\left(y_{i} \mid \phi_{i}, z_{i}\right)=p\left(z_{i}\right) p\left(\phi \mid z_{i}\right) p\left(y_{i} \mid z_{i}\right) .
$$

Note that (4) is true since $Y_{i}$ and $\Phi_{i}\left(Y_{\sim i}\right)$ are independent given $Z_{i}$, i.e., $Y_{i} \rightarrow Z_{i} \rightarrow \Phi_{i}\left(Y_{\sim i}\right)$.

Taking derivative and using $p\left(z_{i} \mid \phi_{i}\right)=p\left(z_{i} \mid y_{\sim i}\right)$, we get

$$
\begin{aligned}
\mathrm{G}_{i}(\mathrm{~h})= & \sum_{z_{i}} p\left(z_{i}\right) \int_{\phi_{i}} p\left(\phi_{i} \mid z_{i}\right) \int_{y_{i}} \frac{\mathrm{d}}{\mathrm{dh}_{i}} p\left(y_{i} \mid z_{i}\right) . \\
& \cdot \log _{2}\left\{\sum_{z_{i}^{\prime}} \frac{p\left(z_{i}^{\prime} \mid y_{\sim i}\right) p\left(y_{i} \mid z_{i}^{\prime}\right)}{p\left(z_{i} \mid y_{\sim i}\right) p\left(y_{i} \mid z_{i}\right)}\right\} \mathrm{d} y_{i} \mathrm{~d} \phi_{i} \\
= & \sum_{z} p(z) \int_{\underline{v}} \mathrm{a}_{i, z}(\underline{v}) \kappa_{i, z}(\underline{v}) \mathrm{d} \underline{v} .
\end{aligned}
$$

where

$$
\begin{aligned}
\kappa_{i, z}(\underline{v}) & =\int_{y_{i}} \frac{\mathrm{d}}{\mathrm{dh}_{i}} p\left(y_{i} \mid z\right) \log _{2}\left\{\frac{\sum_{z^{\prime}} v_{\left[z^{\prime}\right]} p\left(y_{i} \mid z^{\prime}\right)}{v_{[z]} p\left(y_{i} \mid z\right)}\right\} \mathrm{d} y_{i} \\
& =\int_{y_{i}} \frac{\partial}{\partial \epsilon} p\left(y_{i} \mid z\right) \log _{2}\left\{\frac{\sum_{z^{\prime}} v_{\left[z^{\prime}\right]} p\left(y_{i} \mid z^{\prime}\right)}{v_{[z]} p\left(y_{i} \mid z\right)}\right\} \mathrm{d} y_{i} / \frac{\partial \mathrm{h}_{i}}{\partial \epsilon} .
\end{aligned}
$$

Finally, by seeing that

$$
\begin{aligned}
\frac{\partial \mathrm{h}_{i}}{\partial \epsilon} & =\frac{\partial H\left(Z_{i} \mid Y_{i}(\epsilon)\right)}{\partial \epsilon} \\
& =\sum_{z} \int_{y_{i}} p(z) \frac{\partial}{\partial \epsilon} p\left(y_{i} \mid z\right) \log _{2}\left\{\frac{\sum_{z^{\prime}} p\left(z^{\prime}\right) p\left(y_{i} \mid z^{\prime}\right)}{p(z) p\left(y_{i} \mid z\right)}\right\} \mathrm{d} y_{i} .
\end{aligned}
$$

we obtain the result.

Remark 1: For $\sigma=0$ in the AWGN case (or $\epsilon=0$ in erasure noise), $\mathrm{h}=0$ and $\mathrm{a}_{i, z}$ is "delta at $\underline{v}=e_{[z]}$ " where $e_{[z]}$ is the standard basis vector. At this extreme, $\mathrm{G}(0)=0$ since $\kappa_{i, z}(\underline{v})=0$. At the other extreme $\sigma \rightarrow \infty$ (or at $\epsilon=1$ for erasure noise), $\mathrm{h}=H(Z)$ (e.g., 1.5 for the dicode channel) and $\mathrm{G}(\mathrm{h})=1$ since in this case $\mathrm{a}_{i, z}$ is "delta at $v_{\left[z^{\prime}\right]}=p\left(z^{\prime}\right) \forall z^{\prime \prime}$ ".

1) BP-GEXIT curve (with $A W G N$ ): In this section, we are particularly interested in computing the BP-GEXIT function for ISI channels with AWGN. In this case, let $\Phi_{i}^{\mathrm{BP}, \ell}$ denote the extrinsic estimate of $Z_{i}$ at the $\ell$ th round of joint $\mathrm{BP}$ decoding. If $\Phi_{i}^{\mathrm{BP}, \ell}$ is used instead of $\Phi_{i}$ in the above formulas then one has the BP-GEXIT (at the $\ell$ th round) $\mathrm{G}^{\mathrm{BP}, \ell}$ in a similar manner to [21] and the overall BP-GEXIT $G^{\mathrm{BP}}(\mathrm{h})=\lim _{\ell \rightarrow \infty} \mathrm{G}^{\mathrm{BP}, \ell}(\mathrm{h})$. Also, notice that the two extremes in Remark 1 still apply when the BP decoder is used instead of the MAP decoder. 
Next, AWGN implies that $p\left(y_{i} \mid z\right)=\frac{1}{\sqrt{2 \pi \sigma^{2}}} e^{-\frac{\left(y_{i}-z\right)^{2}}{2 \sigma^{2}}}$ and then $\frac{\partial}{\partial \epsilon} p\left(y_{i} \mid z\right)=\left(\left(y_{i}-z\right)^{2}-\sigma^{2}\right) p\left(y_{i} \mid z\right)$. Therefore, the corresponding $i$ th BP-GEXIT is $\mathrm{G}_{i}^{\mathrm{BP}, \ell}(\mathrm{h})=\frac{A}{B}$ where

$$
\begin{gathered}
A=\sum_{z} p(z) \int_{\underline{v}} \mathrm{a}_{i, z}^{\mathrm{BP}, \ell}(\underline{v}) \int_{-\infty}^{\infty} p\left(y_{i} \mid z\right)\left\{\frac{\left(y_{i}-z\right)^{2}}{\sigma^{2}}-1\right\} . \\
\cdot \log _{2}\left\{\sum_{z^{\prime}} \frac{v_{\left[z^{\prime}\right]}}{v_{[z]}} e^{\frac{\left(z^{\prime}-z\right)\left(2 y_{i}-z-z^{\prime}\right)}{2 \sigma^{2}}}\right\} \mathrm{d} y_{i} \mathrm{~d} \underline{v}, \\
B=\sum_{z} p(z) \int_{-\infty}^{\infty} p\left(y_{i} \mid z\right)\left\{\frac{\left(y_{i}-z\right)^{2}}{\sigma^{2}}-1\right\} . \\
\cdot \log _{2}\left\{\sum_{z^{\prime}} \frac{p\left(z^{\prime}\right)}{p(z)} e^{\frac{\left(z^{\prime}-z\right)\left(2 y_{i}-z-z^{\prime}\right)}{2 \sigma^{2}}}\right\} \mathrm{d} y_{i} .
\end{gathered}
$$

In the limit of $\ell \rightarrow \infty$, one can run the DE for ISI channels [6] to obtain the DE-FP and compute the quantities $A$ and $B$ at this FP. With some abuse of notation, let $\mathrm{a}^{(\ell)}, \mathrm{b}^{(\ell)}, \mathrm{c}^{(\ell)}$ and $d^{(\ell)}$ denote the average density of the bit-to-check, check-tobit, bit-to-trellis and trellis-to-bit messages, respectively (see Fig. 1), at iteration $\ell$ with initial values (at $\ell=0$ ) being $\Delta_{0}$, the delta function at 0 . Also, let $\mathrm{n}$ denote the density of channel noise. The DE update equation for joint BP decoding of a general binary-input ISI channels is

$$
\begin{array}{ll}
\mathrm{a}^{(\ell)}=\mathrm{d}^{(\ell-1)} \circledast \lambda\left(\mathrm{b}^{(\ell-1)}\right), & \mathrm{b}^{(\ell)}=\rho\left(\mathrm{a}^{(\ell)}\right), \\
\mathrm{c}^{(\ell)}=L\left(\mathrm{~b}^{(\ell)}\right), & \mathrm{d}^{(\ell)}=\Gamma\left(\mathrm{c}^{(\ell)}, \mathrm{n}\right),
\end{array}
$$

where for a density $\mathrm{x}, \lambda(\mathrm{x})=\sum_{i} \lambda_{i} \mathrm{x}^{\otimes(i-1)}, \rho(\mathrm{x})=$ $\sum_{i} \rho_{i} \mathrm{x}^{\circledast(i-1)}$ and $L(\mathrm{x})=\sum_{i} L_{i} \mathrm{x}^{\otimes i}$. The operators $\otimes$ and $⿴$ are the standard density transformations used in [18, p. 181]. The map $\Gamma(\cdot, \cdot)$ is not easy to compute in closed form for general trellises and often one needs to resort to the Monte Carlo methods (i.e., running the windowed BCJR algorithm with window parameter $W$ on a long enough trellis - see details in [6]) to give the estimates. A similar method was used to upper bound the MAP threshold for turbo codes over BMS channels [22].

The denominator $B$ can be computed either by numerical integration or by Monte Carlo methods. Meanwhile, the numerator $A$ involves in the quantity $v_{[z]}=p\left(Z_{i}=z \mid \mathrm{T}_{i}^{\ell}\right)$ where $\mathrm{T}_{i}^{\ell}$ denotes the computation tree of depth $\ell$, rooted at index $i$, which includes all channel and code constraints associated with $\ell$ iterations of decoding. This computation tree $\mathrm{T}_{i}^{\ell}$ excludes the tree root $y_{i}$ and is implied by the decoding schedule in the DE equation. The quantity $v_{[z]}$, due to complications from the trellis, is not easy to obtain in closed form. However, one can readily compute $v_{[z]}$ as an extra output of the BCJR algorithm (which is already used in DE) using

$$
v_{[z]} \propto \sum_{s_{i}, s_{i-1}: Z_{i}=z} \alpha_{i-1}\left(s_{i-1}\right) \cdot \gamma_{i}\left(s_{i-1}, s_{i}\right) \cdot \beta_{i}\left(s_{i}\right) .
$$

where $\gamma_{i}\left(s_{i-1}, s_{i}\right)$ is probability of the input $x_{i}$ that corresponds to the transition from state $s_{i-1}$ (at time index $i-1$ ) to state $s_{i}$ at (time index $i$ ) given the computation tree $\mathrm{T}_{i}^{\ell}$. Here, $\alpha_{i}(\cdot)$ and $\beta_{i}(\cdot)$ are the standard forward and backward state probabilities in the BCJR algorithm. Note that the scaling constant can be chosen so that $\sum_{z} v_{[z]}=1$.

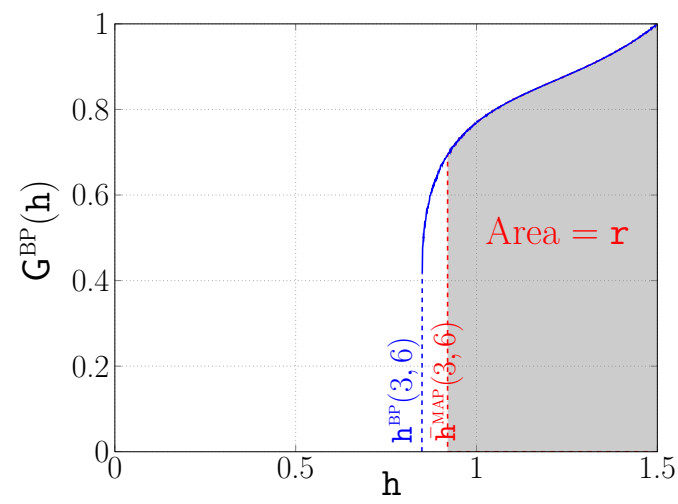

Figure 3. BP-GEXIT curve for a $(3,6)$-regular LDPC code over an AWGN dicode channel. The upper bound $\overline{\mathrm{h}}^{\mathrm{MAP}}$ is obtained by setting the area under the BP-GEXIT curve (the shaded region) equal to the code rate.

\section{B. Upper Bound for the MAP Threshold}

The above-mentioned GEXIT curve naturally obeys the area theorem $\int_{\mathrm{h}^{\mathrm{MAP}}}^{H(Z)} \mathrm{G}(\mathrm{h}) \mathrm{dh}=\int_{0}^{H(Z)} \mathrm{G}(\mathrm{h}) \mathrm{dh}=\mathrm{r}$. Therefore, one can apply the discussed bounding technique, i.e., by finding the largest value $\overline{\mathrm{h}}^{\mathrm{MAP}}$ such that the area under the BP-GEXIT curve equals the code rate,

$$
\int_{\overline{\mathrm{h}}^{\mathrm{MAP}}}^{H(Z)} \mathrm{G}^{\mathrm{BP}}(\mathrm{h}) \mathrm{dh}=\mathrm{r},
$$

to obtain the MAP upper bound $\bar{h}^{\text {MAP }} \geq \mathrm{h}^{\text {MAP }}$.

For example, the BP-GEXIT curve for the $(3,6)$-regular LDPC code over an AWGN dicode channel with $a(D)=$ $(1-D) / \sqrt{2}$ following the analysis in Section III-A is shown in Fig. 3. In this case, $\mathrm{h}^{\mathrm{BP}}(3,6) \approx 0.851$ (the corresponding threshold measured in $\mathrm{dB}$ is $\left.\sigma^{\mathrm{BP}}(3,6) \approx 1.703 \pm 0.001 \mathrm{~dB}\right)$ while $\overline{\mathrm{h}}^{\mathrm{MAP}}(3,6) \approx 0.920$ (or $\bar{\sigma}^{\mathrm{MAP}}(3,6) \approx 0.959 \pm 0.001 \mathrm{~dB}$ ).

\section{Spatially-Coupled Codes on the ISI Channels}

Consider the $(l, r, L)$ spatially-coupled ensemble. For the ISI channels, the DE equation for this ensemble can be obtained from the protograph chain in a similar manner to the case of memoryless channels discussed in [2]. For each $i, j \in[1-\hat{l}, L+\hat{l}]$, let $\mathrm{a}_{i \rightarrow j}^{(\ell)}\left(\right.$ and $\mathrm{b}_{i \leftarrow j}^{(\ell)}$ ) denote the average density of the messages from bit nodes at position $i$ to check nodes at position $j$ (and the other way around) ${ }^{6}$. With all the initial message densities (at $\ell=0$ ) being $\Delta_{0}$, the DE update equation (for all $i \in[1, L]$ ) is

$$
\begin{aligned}
& \mathrm{a}_{i \rightarrow j}^{(\ell)}=\mathrm{d}_{i}^{(\ell-1)} \circledast\left\{\underset{j^{\prime} \in[i-\hat{l}, i+\hat{l}] \backslash j}{\otimes} \mathrm{b}_{i \leftarrow j^{\prime}}^{(\ell-1)}\right\}, \forall j \in[i-\hat{l}, i+\hat{l}], \\
& \mathrm{b}_{i \leftarrow j}^{(\ell)}=\underset{i^{\prime} \in[j-\hat{l}, j+\hat{l}] \backslash i}{\mathrm{a}_{i^{\prime} \rightarrow j}^{(\ell)}, \forall j \in[i-\hat{l}, i+\hat{l}],} \\
& \mathrm{c}_{i}^{(\ell)}=\underset{j^{\prime} \in[i-\hat{l}, i+\hat{l}]}{\circledast} \mathrm{b}_{i \leftarrow j^{\prime}}^{(\ell)}, \\
& \mathrm{d}_{i}^{(\ell)}=\Gamma\left(\mathrm{c}_{i}^{(\ell)}, \mathrm{n}\right)
\end{aligned}
$$

where $\otimes_{j \in\left\{j_{1}, \ldots, j_{t}\right\}} \mathrm{x}_{j}$ and $\Theta_{i \in\left\{i_{1}, \ldots, i_{t}\right\}} \mathrm{x}_{i}$ denote the operations $\mathrm{x}_{j_{1}} \otimes \mathrm{x}_{j_{2}} \otimes \ldots \otimes \mathrm{x}_{j_{t}}$ and $\mathrm{x}_{i_{1}}$ 因 $\mathrm{x}_{i_{2}}$ 因... $⿴ \mathrm{x}_{i_{t}}$, respectively.

\section{Simulation Results}

We start with the $(l, r, L)$ circular ensemble obtained by considering all the positions $i>L$ of the protograph chain

\footnotetext{
${ }^{6}$ For $i \notin[1, L]$, set $\mathrm{a}_{i \rightarrow j}^{(\ell)}=\Delta_{+\infty}$, the delta function at $+\infty$.
} 


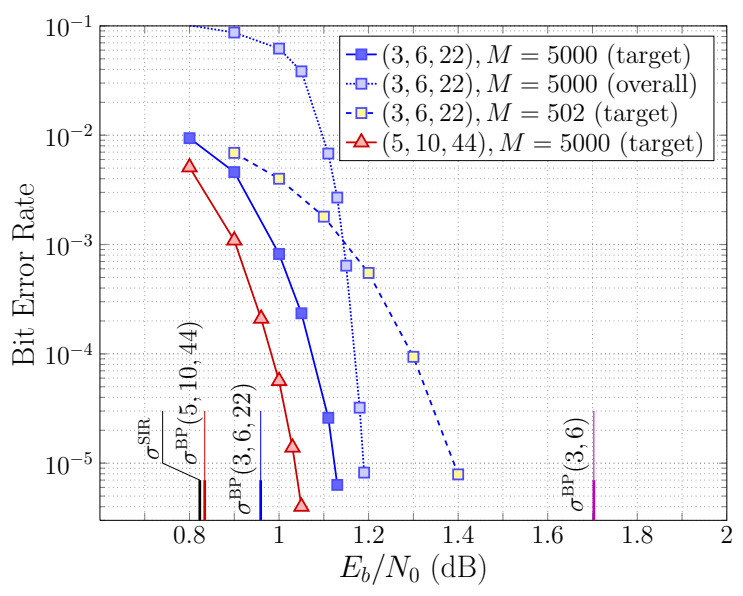

Figure 4. BER and BP thresholds for the $(3,6)$-regular, $(3,6,22)$ and $(5,10,44)$ spatially-coupled ensembles over the AWGN dicode channel.

to be the same as positions $i-L$ (similar to [5]). The order of bit transmissions is "left to right" in each length- $L$ row and then start with the next row (in a total of $M$ rows, see Fig. 2). The $I \triangleq \max (\nu, l-1)$ first bits in each row are known. These bits will "break" the circular ensemble into the $(l, r, L-$ $I$ ) ensemble and also serve as the pilot bits to fix the trellis state. Due to this fixing, one only needs to run the BCJR independently in each row and in a parallel manner [10], [11].

In our experiments, we conduct simulations over the AWGN dicode channel with $a(D)=(1-D) / \sqrt{2}$ and memory $\nu=1$. First, we use the DE in Sec. III-C to compute the BP thresholds of the spatially-coupled coding scheme. The results in Fig. 4 reveal that $\sigma^{\mathrm{BP}}(3,6,22)$ is roughly $0.959 \pm 0.001 \mathrm{~dB}$ and approximately the same as $\sigma^{\mathrm{BP}}(3,6,44)$ whose rate loss is smaller. Notice that this is also roughly $\bar{\sigma}^{\mathrm{MAP}}(3,6)$ - the MAP threshold estimate of the underlying $(3,6)$-regular ensemble, obtained by the bounding technique, and is a significant improvement over $\sigma^{\mathrm{BP}}(3,6) \approx 1.703 \pm 0.001 \mathrm{~dB}$. This suggests that threshold saturation occurs for regular ensembles. Since MAP decoding of regular ensembles can achieve the SIR [15], if threshold saturation occurs, one can universally approach the SIR of general ISI channels using coupled codes with joint iterative decoding. To support this, one can also see that for the $(5,10,44)$ ensemble of the same rate as the $(3,6,22)$ one, the threshold $\sigma^{\mathrm{BP}}(5,10,44) \approx 0.834 \pm 0.001 \mathrm{~dB}$ gets very close to the signal-to-noise ratio (SNR) corresponding to the SIR $\left(\sigma^{\mathrm{SIR}} \approx 0.823 \pm 0.001 \mathrm{~dB}\right.$ using the numerical method in [16], [17]). Although only simulations with the dicode channel are shown, the overall method is readily applicable to channels with higher memory.

Also shown in Fig. 4 is the bit error rate (BER) versus SNR plot for the ensembles derived from the $(l, r, L)$ circular ensembles of finite $M=502$ and $M=5000$. For each simulation, we use $l_{\text {outer }}=20$ channel updates and between two such channel updates, we run $l_{\text {inner }}=5 \mathrm{BP}$ iterations on the code part alone. The curves labeled "target" is the BER for the bits at position $I+1$ (right after the known bits) in the coupled chain while the curve labeled "overall" is the overall BER for all the positions $[I+1, L]$ together. One might expect that the "overall" BER will get closer to the "target" BER for large enough $M$ and large enough number of iterations using
Table I

THRESHOLD ESTIMATES OF $(l, r)$-REgUlAR ENSEMBLES OVER THE DEC AND DICODE AWGN CHANNEL. FOR AWGN NOISE, THE THRESHOLDS ARE MEASURED IN $\mathrm{dB}$.

\begin{tabular}{|c||c|c|c|c|c|c|}
\hline \multicolumn{1}{|c||}{$\begin{array}{c}(l, r)- \\
\text { regular }\end{array}$} & \multicolumn{3}{c|}{ DEC } & \multicolumn{3}{c|}{ Dicode AWGN } \\
\cline { 2 - 7 } & $\epsilon^{\mathrm{BP}}$ & $\bar{\epsilon}^{\mathrm{MAP}}$ & $\epsilon^{\mathrm{SIR}}$ & $\sigma^{\mathrm{BP}}$ & $\bar{\sigma}^{\mathrm{MAP}}$ & $\sigma^{\mathrm{SIR}}$ \\
\hline \hline$(3,6)$ & 0.5689 & 0.6387 & 0.6404 & 1.073 & 0.959 & 0.823 \\
\hline$(5,10)$ & 0.4647 & 0.6404 & 0.6404 & 3.032 & 0.834 & 0.823 \\
\hline
\end{tabular}

an induction argument. From Fig. 4, one can also observe that the "overall" BER for $(3,6,22)$ and $M=5000$ keeps getting "closer" to the "target" BER as SNR slightly increases. Those BER curves are significantly improved with respect to $\epsilon^{\mathrm{BP}}(3,6)$ - the $\mathrm{BP}$ threshold for the underlying $(3,6)$-regular ensemble.

\section{ISI Channels with ERASURE Noise: The DEC}

In this section, we briefly discuss threshold saturation on the DEC. For this channel, the GEXIT curves mentioned above becomes identical (after scaling) to the EXIT curves derived in [23]. From these curves, one can also obtain a numerically tight upper bound on the MAP threshold of the underlying ensemble and observe the threshold saturation effect.

\section{A. Upper Bound on the MAP Threshold}

For the DEC and regular LDPC ensembles, the MAP upper bound was first considered in [23]. In a recent report [24], the authors further provide a closed-form solution for the BPEXIT and extended BP (EBP) EXIT curves that can quickly gives an upper bound $\bar{\epsilon}^{\mathrm{MAP}}$ on the MAP threshold by setting the area under the EBP curve (the shaded area in Fig. 5) equal to the code rate. The tightness of the bounding technique is strongly suggested by a counting argument and for the $(l, r)$-regular ensemble, this upper bound can be shown to quickly approach the erasure rate associated with the SIR when increasing $l, r$ such that the code rate $\mathrm{r}$ is fixed (see arguments in [24] and facts in Table I).

\section{B. Spatially-Coupled Codes for the DEC}

Consider the $(l, r, L, w)$ spatially-coupled ensemble. We also follow the DE equation discussed in [13] to compute the BP thresholds of the coupled ensembles. The main difference is that we use the correct EBP-EXIT curves with their operational meaning instead of the EXIT-like curves used in [13]. Let $x_{i}^{(\ell)}$ denote the expected erasure rate at iteration $\ell$ from bit nodes at position $i$ to check nodes where for $i \notin[1, L]$, one sets $x_{i}^{(\ell)}=0$. To compute both the stable and unstable FPs of DE, one can use the fixed entropy DE procedure outlined in [21, Sec. VIII] where the normalized entropy of a constellation $\underline{x}^{(\ell)}=\left(x_{1}^{(\ell)}, \ldots, x_{L}^{(\ell)}\right)$, which is defined as $\chi\left(\underline{x}^{(\ell)}\right)=\frac{1}{L} \sum_{i=1}^{L} x_{i}^{(\ell)}$, is kept constant at every iteration by varying the channel parameter. With each FP $\underline{x}$ obtained, one obtains the EBP-EXIT value of the spatially-coupled ensemble as $\frac{1}{L} \sum_{i=1}^{L} h^{\mathrm{EBP}}\left(x_{i}\right)$ where $h^{\mathrm{EBP}}(\cdot)$ is the EBP-EXIT function defined in [24].

The threshold saturation effect of coupling can be nicely seen by plotting the EBP-EXIT curves for the uncoupled and coupled codes. For example, Fig. 5 shows the EBP curves for the $(3,6, L, 5)$ ensembles with various $L$ along with the 


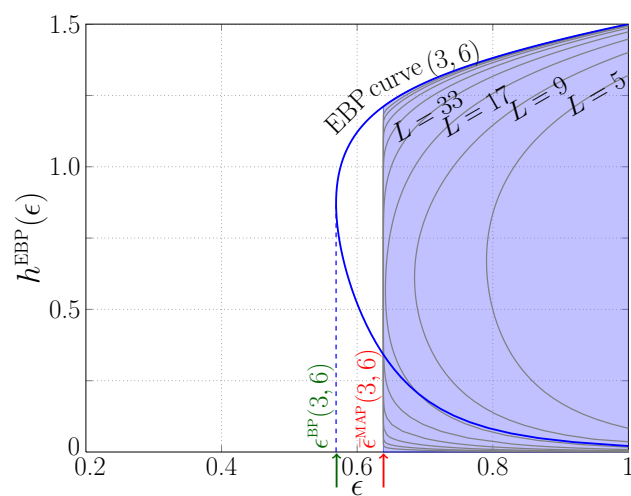

Figure 5. EBP-EXIT curves for $(3,6, L, 5)$ with $L=2 \hat{L}+1$ where $\hat{L}=$ $2,4,8,16,32,64,128,246$ over the DEC. As $L$ grows larger, the rate loss becomes negligible and the curves keep moving left, but they saturate at the MAP threshold of the underlying regular ensemble.

EBP curve of the underlying $(3,6)$-regular ensemble. From the EBP curves, one can determine $\epsilon^{\mathrm{BP}}(3,6) \approx 0.56892$ and $\bar{\epsilon}^{\mathrm{MAP}}(3,6) \approx 0.63866$. The BP thresholds of spatially-coupled ensembles for small $L$ due to rate-loss can have larger values, e.g., $\epsilon^{\mathrm{BP}}(3,6,17,6) \approx 0.64170>\bar{\epsilon}^{\mathrm{MAP}}(3,6)$. However, for a wide range of $L$, i.e., $L=33,65,129,257,513$, we observe that $\epsilon^{\mathrm{BP}}(3,6, L, 5) \approx 0.63866$ which is essentially $\bar{\epsilon}^{\mathrm{MAP}}(3,6)$ while the rate loss gradually becomes insignificant. In [13] , Kudekar and Kasai provided a similar plot based on the EXITlike function borrowed from the EXIT function of the BEC; though the picture is similar, it is not associated with an area theorem for the DEC. Instead, we use a proper EXIT function $h^{\mathrm{EBP}}$ and obtain the MAP threshold estimate $\bar{\epsilon}^{\mathrm{MAP}}$.

\section{CONCLUDING REMARKS}

In this paper, we consider binary communication over the ISI channels and numerically show that the threshold saturation effect occurs on both the DEC and dicode channel with AWGN. To do this, we construct the (G)EXIT curves that satisfy the area theorem and obtain an upper bound on the threshold of the MAP decoder. The upper bound is conjectured to be tight and, for the DEC, a numerical evidence can be shown to strongly support this conjecture. The observed threshold saturation effect is valuable because by changing the underlying regular LDPC ensemble combined with the results of [15], it is shown that the joint BP decoding of spatially-coupled codes can universally approach the SIR of the ISI channels. Also, the convolutional structure of the codes allows one to consider a windowed decoder similar to the one discussed in [25], [26]. All of these properties suggest that spatially-coupled codes may be competitive in practice for systems with ISI.

\section{REFERENCES}

[1] J. Felstrom and K. S. Zigangirov, "Time-varying periodic convolutional codes with low-density parity-check matrix," IEEE Trans. Inform. Theory, vol. 45 , no. 6 , pp. 2181-2191, 1999.

[2] M. Lentmaier, A. Sridharan, D. J. Costello, and K. S. Zigangirov, "Iterative decoding threshold analysis for LDPC convolutional codes," IEEE Trans. Inform. Theory, vol. 56, no. 10, pp. 5274-5289, Oct. 2010.

[3] S. Kudekar, T. Richardson, and R. Urbanke, "Threshold saturation via spatial coupling: Why convolutional LDPC ensembles perform so well over the BEC," IEEE Trans. Inform. Theory, vol. 57, no. 2, pp. 803-834, 2011.
[4] M. Lentmaier and G. Fettweis, "On the thresholds of generalized LDPC convolutional codes based on protographs," in Proc. IEEE Int. Symp. Inform. Theory, Austin, TX, 2010, pp. 709-713.

[5] S. Kudekar, C. Méasson, T. Richardson, and R. Urbanke, "Threshold saturation on BMS channels via spatial coupling," in Proc. Int. Symp. on Turbo Codes \& Iterative Inform. Proc., Sept. 2010, pp. 309-313.

[6] A. Kavčić, X. Ma, and M. Mitzenmacher, "Binary intersymbol interference channels: Gallager codes, density evolution and code performance bounds," IEEE Trans. Inform. Theory, vol. 49, no. 7, pp. 1636-1652, July 2003.

[7] B. M. Kurkoski, P. H. Siegel, and J. K. Wolf, "Joint message-passing decoding of LDPC codes and partial-response channels," IEEE Trans. Inform. Theory, vol. 48, no. 6, pp. 1410-1422, June 2002.

[8] H. D. Pfister and P. H. Siegel, "Joint iterative decoding of LDPC codes and channels with memory," in Proc. 3rd Int. Symp. on Turbo Codes \& Related Topics, Brest, France, Sept. 2003, pp. 15-18.

[9] N. Varnica and A. Kavčić, "Optimized low-density parity-check codes for partial response channels," IEEE Commun. Letters, vol. 7, no. 4, pp. $168-170,2003$

[10] K. R. Narayanan and N. Nangare, "A BCJR-DFE based receiver for achieving near capacity performance on inter symbol interference channels," in Proc. 43rd Annual Allerton Conf. on Commun., Control, and Comp., Monticello, IL, Oct. 2004, pp. 763-772.

[11] J. B. Soriaga, H. D. Pfister, and P. H. Siegel, "Determining and approaching achievable rates of binary intersymbol interference channels using multistage decoding," IEEE Trans. Inform. Theory, vol. 53, no. 4, pp. 1416-1429, April 2007.

[12] H. D. Pfister and P. H. Siegel, "Joint iterative decoding of LDPC codes for channels with memory and erasure noise," IEEE J. Select. Areas Commun., vol. 26, no. 2, pp. 320-337, Feb. 2008.

[13] S. Kudekar and K. Kasai, "Threshold saturation on channels with memory via spatial coupling," in Proc. IEEE Int. Symp. Inform. Theory, St. Petersburg, Russia, July 2011, pp. 2562-2566.

[14] H. D. Pfister, "On the capacity of finite state channels and the analysis of convolutional accumulate- $m$ codes," Ph.D. dissertation, University of California, San Diego, La Jolla, CA, USA, March 2003.

[15] J. H. Bae and A. Anastasopoulos, "Capacity-achieving codes for finitestate channels with maximum-likelihood decoding," IEEE J. Select. Areas Commun., vol. 27, no. 6, pp. 974-984, Aug. 2009.

[16] D. Arnold and H. Loeliger, "On the information rate of binary-input channels with memory," in Proc. IEEE Int. Conf. Commun., Helsinki, Finland, June 2001, pp. 2692-2695.

[17] H. D. Pfister, J. B. Soriaga, and P. H. Siegel, "On the achievable information rates of finite state ISI channels," in Proc. IEEE Global Telecom. Conf., San Antonio, Texas, USA, Nov. 2001, pp. 2992-2996.

[18] T. J. Richardson and R. L. Urbanke, Modern Coding Theory. Cambridge University Press, 2008.

[19] C. Douillard, M. Jézéquel, C. Berrou, A. Picart, P. Didier, and A. Glavieux, "Iterative correction of intersymbol interference: Turbo equalization," Eur. Trans. Telecom., vol. 6, no. 5, pp. 507-511, Sept. Oct. 1995.

[20] J. Hou, P. H. Siegel, L. B. Milstein, and H. D. Pfister, "Capacityapproaching bandwidth-efficient coded modulation schemes based on low-density parity-check codes," IEEE Trans. Inform. Theory, vol. 49, no. 9, pp. 2141-2155, Sept. 2003.

[21] C. Méasson, A. Montanari, T. Richardson, and R. Urbanke, "The generalized area theorem and some of its consequences," IEEE Trans. Inform. Theory, vol. 55, no. 11, pp. 4793-4821, Nov. 2009.

[22] - "Maximum a posteriori decoding and turbo codes for general memoryless channels," in Proc. IEEE Int. Symp. Inform. Theory, Adelaide, Australia, 2005, pp. 1241-1245.

[23] C. Wang and H. D. Pfister, "Upper bounds on the MAP threshold of iterative decoding systems with erasure noise," in Proc. Int. Symp. on Turbo Codes \& Related Topics, Lausanne, Switzerland, Sept. 2008, pp. $7-12$.

[24] P. S. Nguyen, A. Yedla, H. D. Pfister, and K. R. Narayanan, "Spatiallycoupled codes and threshold saturation on intersymbol-interference channels," 2012, to be submitted to IEEE Trans. on Inform. Theory, [Online]. Available: http://arxiv.org/abs/1107.3253.

[25] A. R. Iyengar, M. Papaleo, P. H. Siegel, J. K. Wolf, A. VanelliCoralli, and G. E. Corazza, "Windowed decoding of protographbased LDPC convolutional codes over erasure channels," Oct. 2010, submitted to IEEE Trans. on Inform. Theory [Online]. Available: http://arxiv.org/abs/1010.4548.

[26] A. R. Iyengar, P. H. Siegel, R. L. Urbanke, and J. K. Wolf, "Windowed decoding of spatially coupled codes," in Proc. IEEE Int. Symp. Inform. Theory, St. Petersburg, Russia, July 2011, pp. 2552-2556. 